EPJ manuscript No.

(will be inserted by the editor)

\title{
Polarization observables in high-energy deuteron photo- disintegration within the Quark-Gluon Strings Model ${ }^{\star}$
}

\author{
V.Yu. Grishina ${ }^{a}$, L.A. Kondratyuk ${ }^{b, c}$, W. Cassing ${ }^{d}$, E. De Sanctis ${ }^{e}$, M. Mirazita $^{e}$, F. Ronchetti ${ }^{e}$ and P. Rossi $^{e}$ \\ ${ }^{a}$ Institute for Nuclear Research, 60th October Anniversary Prospect 7A, 117312 Moscow, Russia \\ ${ }^{b}$ Institute for Theoretical and Experimental Physics, B. Cheremushkinskaya 25, 117259 Moscow, Russia \\ ${ }^{c}$ IKP, Forschungszentrum Jülich, D-52425 Jülich,Germany \\ ${ }^{d}$ Institute for Theoretical Physics, University of Giessen, Heinrich-Buff-Ring 16, D-35392 Giessen, Germany \\ ${ }^{e}$ Frascati National Laboratories, INFN, CP 13, via E. Fermi, 40; I-00044, Frascati, Italy
}

Received: date / Revised version: date

\begin{abstract}
Deuteron two-body photodisintegration is analysed within the framework of the Quark-Gluon Strings Model. The model describes fairly well the recent experimental data from TJNAF in the few GeV region. Angular distributions at different $\gamma$-energies are presented and the effect of a forward-backward asymmetry is discussed. New results from the QGSM for polarization observables from $1.5-6 \mathrm{GeV}$ are presented and compared with the available data.
\end{abstract}

PACS. 13.40.-f Electromagnetic processes and properties - 25.20.-x Photonuclear reactions

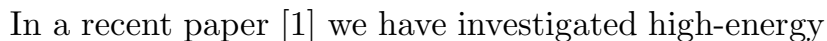
deuteron photodisintegration within the framework of the Quark-Gluon Strings Model (QGSM). The QGSM - proposed by Kaidalov [2] - is based on two ingredients: i) a topological expansion in QCD and ii) a space-time picture of the interactions between hadrons that takes into account the confinement of quarks. In a more general sense the QGSM can be considered as a microscopic (nonperturbative) model of Regge phenomenology for the analysis of exclusive and inclusive hadron-hadron and photohadron reactions on the quark level. Within the QGSM the deuteron photodisintegration amplitude $T(\gamma d \rightarrow p n)$ can be described in first approximation by the planar graph with three valence quark exchange in $t$ (or $u$ )-channels, which corresponds to a nucleon Regge trajectory. The intermediate $s$ channel will consist of a $6 q$ string (or color tube) with $1 q$ and $5 q$ states at the ends. Assuming that all the intermediate quark clusters have minimal spins and the $s$ channel helicities in the quark-hadron and hadronquark transition amplitudes are conserved, we can reconstruct the spin structure of the amplitude $T(\gamma d \rightarrow p n)$ as [1]

$$
\begin{aligned}
& \left\langle p_{3}, \lambda_{p} ; p_{4}, \lambda_{n}|\hat{T}(s, t)| p_{2}, \lambda_{d} ; p_{1}, \lambda_{\gamma}\right\rangle= \\
& \quad \bar{u}_{\lambda_{p}}\left(p_{3}\right) \hat{\epsilon}_{\lambda_{\gamma}}\left(A(s, t) \hat{p}_{3}+B(s, t) m\right) \hat{\epsilon}_{\lambda_{d}} v_{\lambda_{n}}\left(p_{4}\right) .
\end{aligned}
$$

The ratio $R=B(s, t) / A(s, t)$ is fixed by the model to the interval $R=1 \div 2$; calculations will be presented for the limiting values $R=1$ and $R=2$ (see below).

\footnotetext{
* Supported by DFG, RFFI and Forschungszentrum Jülich.
}

In Ref. [1] we have analyzed deuteron photodisintegration within the framework of the QGSM employing nonlinear Regge trajectories. Parameters have been fixed by a previous analysis of $p p$ data (i.e. the reaction $p p \rightarrow d \pi^{+}$) and the TJNAF data at $\Theta_{\text {c.m. }}=36^{\circ}$. We have found that the QGSM provides a reasonable description of all new TJNAF data [3]-[6] on deuteron photodisintegration at large momentum transfer $t$ and that the energy dependence of $\mathrm{d} \sigma / \mathrm{d} t$ at $\Theta_{\text {c.m. }}=36 \div 90^{\circ}$ gives new evidence for a nonlinearity of the Regge trajectory $\alpha_{N}(t)$. The best agreement with the data can be achieved using the - QCD motivated - logarithmic form of the Regge trajectory [1],

$$
\alpha_{N}(t)=\alpha_{N}(0)-(\gamma \nu) \ln \left(1-t / T_{B}\right) .
$$

Evidently the QGSM predicts that $d \sigma / d t$ at fixed c.m. angles will decrease faster than any finite power of $s$ and at sufficiently large energies the perturbative regime will become dominant. Therefore, it is very important to have new data at larger energies to further check the energy behaviour of $d \sigma / d t$ as obtained from the QGSM.

We have also investigated the angular dependence of the cross section at different energies. In Fig. 11 we present the angular dependence of $d \sigma / d t \cdot s^{11}$ at two energies $\left(E_{\gamma}\right.$ $=1.6$ and $3.98 \mathrm{GeV}$ ) for the logarithmic Regge trajectory (2). The two dashed curves have been calculated assuming isovector photon dominance [1]. In this case we get a forward-backward symmetry of the differential cross section. At $1.6 \mathrm{GeV}$ the calculated angular distribution has a dip for $\Theta_{\text {c.m. }}=0^{\circ}$ and $180^{\circ}$ which is related to the choice of the ratio $R=2$. This dip is absent for $R=1$. The 

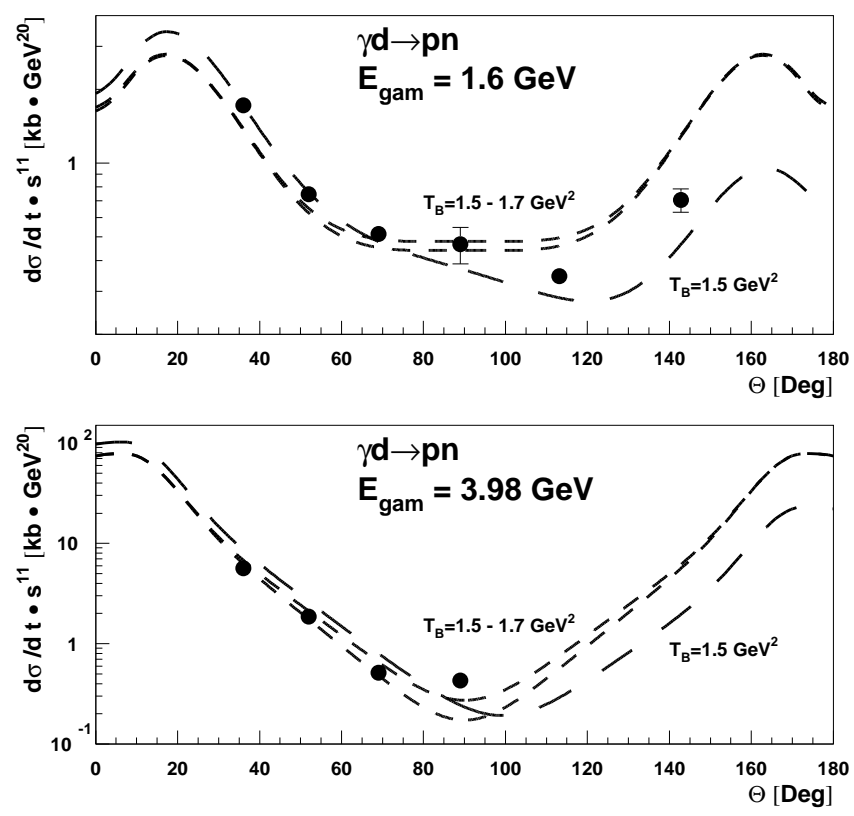

Fig. 1. Differential cross section for the reaction $\gamma d \rightarrow p n$ (multiplied by $s^{11}$ ) as a function of the c. m. angle for $E_{\gamma}=$ $1.6 \mathrm{GeV}$ and $3.98 \mathrm{GeV}$ 1]. The experimental data are from Ref. [6]. The dashed curves are calculated within the QGSM with logarithmic Regge trajectories (2) using $T_{B}=1.5$ and $1.7 \mathrm{GeV}^{2}$ (cf. 11). The long-dashed curve presents the result of calculations which take into account the interference of the isoscalar and isovector parts of the $\gamma d \rightarrow p n$ amplitude (see text).

long-dashed curves in Fig. 1 take into account the forwardbackward asymmetry as discussed below.

We recall, that a forward-backward asymmetry arises from the interference of two amplitudes which describe the contribution of isovector ( $\rho$ like) and isoscalar ( $\omega$ like) photons. In this case the differential cross section can be written as

$$
\begin{aligned}
& \frac{d \sigma_{\gamma d \rightarrow p n}^{\rho+\omega}}{d t}= \\
& \frac{1}{64 \pi s} \frac{1}{\left(p_{\gamma}^{\mathrm{cm}}\right)^{2}} \mid\left\langle\lambda_{p} ; \lambda_{n}\left|\hat{T}^{\rho}(s, t)+\hat{T}^{\omega}(s, t)\right| \lambda_{d} ; \lambda_{\gamma}\right\rangle- \\
& \left.\left\langle\lambda_{p} ; \lambda_{n}\left|\hat{T}^{\rho}(s, t)-\hat{T}^{\omega}(s, t)\right| \lambda_{d} ; \lambda_{\gamma}\right\rangle\right|^{2}
\end{aligned}
$$

Using the vector dominance model (VDM) we adopt

$$
\hat{T}^{\omega}(s, t)=\hat{T}^{\rho}(s, t) / \sqrt{8}, \quad \hat{T}^{\omega}(s, u)=\hat{T}^{\rho}(s, u) / \sqrt{8} .
$$

The data in Fig. 1 at $1.6 \mathrm{GeV}$ provide evidence for a forward-backward asymmetry because the differential cross section at backward angles is smaller than for the corresponding angles in the forward region. The predictions of the QGSM model with $\rho-\omega$ interference are in qualitative agreement with the published data [6](long-dashed lines). They are also in good agreement with the new preliminary data from the CLASS collaboration at TJNAF [4,5].

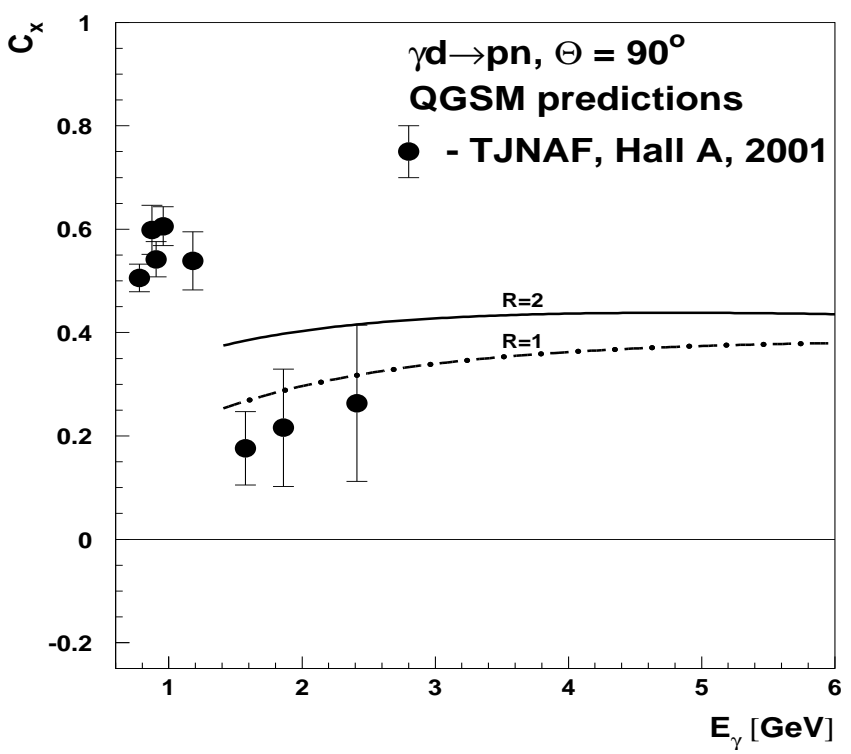

Fig. 2. Polarization transfer $C_{x}$ for circularly polarized photons. The solid and dash-dotted curves correspond to $R=2$ and 1, respectively. The experimental data are from [7] and have been corrected for spin rotation due to the lab. - c.m. transformation.

Complementary information to the differential cross sections is provided by polarization observables that should give important tests of nonperturbative calculations in the intermediate energy regime. Data for recoil polarizations have been published recently [7]. Existing MesonBaryon Models (MBM) fail to describe the data for the induced polarizations, which are surprisingly small for energies above about $1 \mathrm{GeV}$. Moreover, the polarization transfer data are inconsistent with hadron helicity conservation (HHC), which is generally expected for PQCD.

We present here the polarization observables as calculated within the QGSM. For the definitions of these observables in terms of helicity amplitudes we refer the reader to Ref. [8]. We find that

i) the induced polarization $P_{y}$ vanishes at $\Theta_{c . m} .=90^{\circ}$, but is different from 0 for $\Theta_{\text {c.m. }} \neq 90^{\circ}$;

ii) the polarization transfers $C_{x}$ and $C_{z}$ for circularly polarized photons do not vanish at $\Theta_{\text {c.m. }}=90^{\circ}: C_{x} \simeq$ $0.25 \div 0.35$ (Fig. 2) and $C_{z} \simeq-0.1 \div-0.2$ at $1.5-2.5 \mathrm{GeV}$ (Fig. 3) ;

iii) the polarized photon asymmetry $\Sigma$ is about 0.7 at $\Theta_{c . m .}=90^{\circ}$ and $E_{\gamma}=1.5 \mathrm{GeV}$ (Fig. 1 ) and drops smoothly with $E_{\gamma}$.

We observe that for $E_{\gamma} \geq 1.5 \mathrm{GeV}$ the calculated values of $C_{x}$ and $C_{z}$ are in agreement with the new TJNAF data. We note that the term $\sim B(s, t)$ in (1) violates chirality; consequently our results for $C_{x}$ and $C_{z}$ are different from HHC prediction $\left(C_{x}=C_{z}=0\right)$, which is an essential property of PQCD. According to Brodsky and Hiller [10] we should have $\lambda_{d}=\lambda_{p}+\lambda_{n}$ within PQCD independently of $\lambda_{\gamma}$. Assuming that - in the scaling limit the transverse deuteron helicities are suppressed as com- 


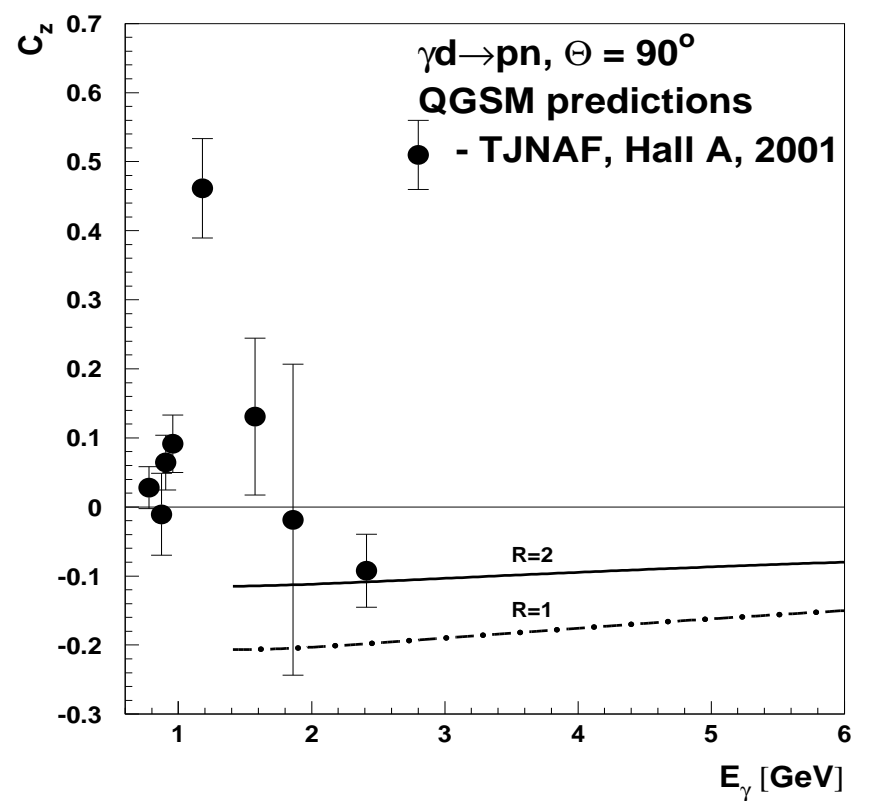

Fig. 3. Polarization transfer $C_{z}$ for circularly polarized photons. The solid and dash-dotted curves correspond to $R=2$ and 1 , respectively. The experimental data are taken from [7] and have been corrected for spin rotation due to the lab. - c.m. transformation.

pared to the longitudinal ones the asymmetry for linearly polarized photons

$$
\Sigma(\theta)=\left(d \sigma_{\|}-d \sigma_{T}\right) /\left(d \sigma_{\|}+d \sigma_{T}\right)
$$

should at $90^{\circ}$ approach the value [11:

$$
\Sigma \simeq-2 \operatorname{Re}\left(T_{+-}^{10} T_{-+}^{10 *}\right) /\left(\left|T_{+-}^{10}\right|^{2}+\mid\left(\left.T_{-+}^{10}\right|^{2}\right) .\right.
$$

Nagornyi et al. 11 predicted - using the axial symmetry $T_{+-}^{10}\left(90^{\circ}\right)=T_{-+}^{10}\left(90^{\circ}\right)$ - that $\Sigma\left(90^{\circ}\right)$ should approach -1 . We note, however, that the condition $T_{+-}^{10}\left(90^{\circ}\right)=$ $T_{-+}^{10}\left(90^{\circ}\right)$ is valid only for 'isoscalar' photons, where the isospin function is antisymmetric. But in case of 'isovector' photons the isospin function is symmetric and due to the Pauli principle we have $T_{+-}^{10}\left(90^{\circ}\right)=-T_{-+}^{10}\left(90^{\circ}\right)$. Furthermore, according to the VDM the isovector photon couples to hadrons more strongly that the isoscalar photon. Thus one expects that in case of $\mathrm{HHC}$ the polarization $\Sigma\left(90^{\circ}\right)$ should not be very different from +1 .

As seen from Fig. 4 the QGSM predicts a slow decrease of $\Sigma\left(90^{\circ}\right)$ with photon energy from $0.7-0.8$ at 1.5 $\mathrm{GeV}$ to $0.4-0.5$ at $5-6 \mathrm{GeV}$. For photon energies below 1.5 $\mathrm{GeV}$ the polarizations $C_{x}$ and $C_{z}$ show quasi-resonance structures at $\sqrt{s}=2.7 \mathrm{GeV}$ and $\sqrt{s}=2.9 \mathrm{GeV}$, respectively. Such resonance structures are conceptually out of the range of the QGSM. It is interesting to note that there are similar structures at exactly the same $\sqrt{s}$ in $\Delta \sigma_{L}$ for elastic $p p$ scattering [12]. We speculate that these quasiresonance structures might be related to the thresholds $\rho N N$ and $\rho N \Delta$.

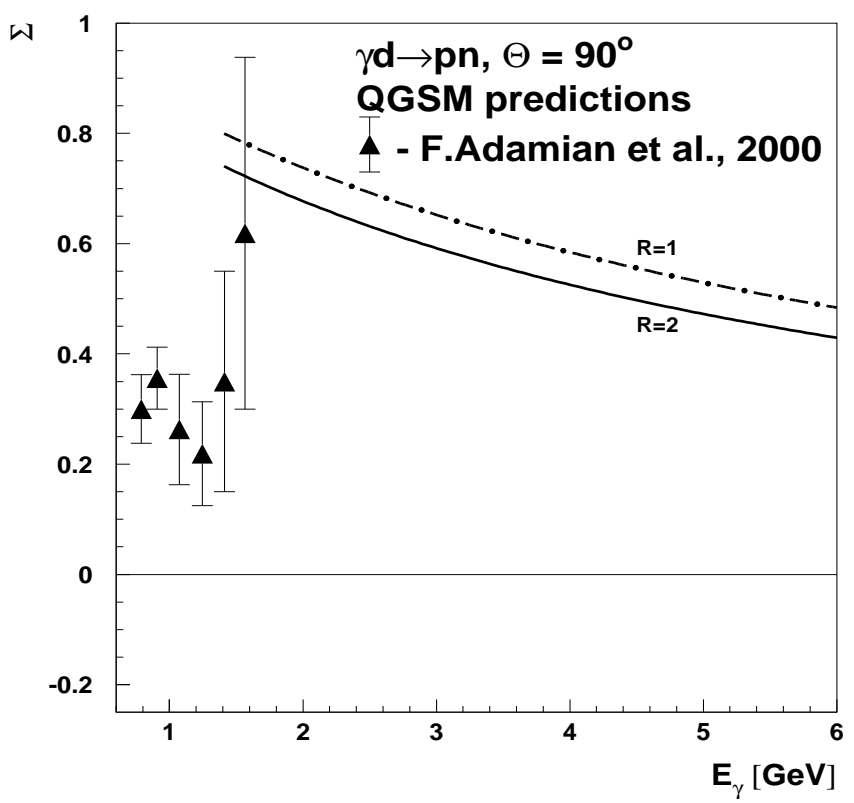

Fig. 4. The asymmetry $\Sigma\left(90^{\circ}\right)$ for linearly polarized photons as a function of the photon energy. The solid and dash-dotted curves correspond to $R=2$ and 1 , respectively.

In summarizing this contribution we have presented angular distributions at different $\gamma$-energies $(1.68 \mathrm{GeV}$ and $3.98 \mathrm{GeV}$ ) for deuteron photodisintegration within the QGSM and discussed the effect of a forward-backward asymmetry. In addition to Ref. [1] new results from the QGSM for polarization observables from $1.5-6 \mathrm{GeV}$ (cf. Figs. 2 - 4) have been calculated and compared to the available data.

We are grateful to Ronald Gilman for sending us the experimental data on the polarizations $C_{x}$ and $C_{z}$ corrected for spin rotation due to the lab. - c.m. transformation.

\section{References}

1. V.Yu. Grishina et al., Eur.Phys.J. A 10, 355 (2001).

2. A.B. Kaidalov, Z. Phys C 12, 63 (2001).

3. E.C. Schulte et al., Phys. Rev. Lett. 87, 102302-1 (2002).

4. M. Mirazita, Proc. of the XL Intern. Winter Meeting on Nuclear Physics, Bormio, January 21-26, 2002; hep$\mathrm{ph} / 0206213$.

5. F. Ronchetti, this conference.

6. C. Bochna et al., Phys. Rev. Lett. 81, 4576 (1998).

7. K. Wijesooriya et al., Phys. Rev. Lett. 86, 2975 (2001).

8. V.P. Barannik et al., Nucl. Phys. A 451, 751 (1986).

9. F. Adamian et al., Eur.Phys.J. A 8, 423 (2000).

10. S.J. Brodsky and J.R. Hiller, Phys. Rev. C 28, 475 (1983).

11. S.I. Nagornyi, Yu. A. Kasatkin, and I.K. Kirichenko, Sov. J. Nucl. Phys. 55, 189 (1992).

12. I.P. Auer et al., Phys. Rev. Lett. 62, 2649 (1989). 